\title{
Simultaneous implementation of food regimens may contain pandemics
}

Chanyuk Lam, Yuhan Lin, Lipeng Liao, Man Tang, Min Fu, Qiuyun Liu凹

School of Life Sciences, Sun Yat-sen University, Guangzhou 510275, China.

$\triangle$ Correspondence: Qiuyun Liu, Ph.D, School of Life Sciences, Sun Yat-sen University, Guangzhou 510275, China. Tel: [+86 (020) 84110296] (lsslqy@mail.sysu.edu.cn). 


\begin{abstract}
Calorie restriction limits the intake of amino acids. Various food regimens can restrict the intake of essential amino acids or all amino acids. To contain pandemics, multiple episodes of 1 to 3 days of simultaneous fasting with modest boiled rice water or fruit juice can be employed, separated by pure starch-based diet or rice starch-based diet with moderate fruit juice intake. Unlike face masks with only prophylactic role, dietary regimens boast dual effects: preventive value and marked reduction of severe cases and mortality.
\end{abstract}

KEYWORDS: calorie restriction; simultaneous fasting; COVID-19; SARS-CoV-2 
Calorie restriction has been demonstrated to extend lifespan, first recognized during the Great Depression in America in the 1930s. However, the underlying mechanism has been a subject of heated debate. Fasting was documented in the Chinese classic A Dream of Red Mansions and British classic literature to cope with epidemics. Yet, the underpinnings for such strategies are still under investigation. SARS and SARS-CoV-2 proteins are overrepresented with valine plus glycine residues (1-2), which are implicated in calcium-binding, the formation of stressful calcium oxalate and cell senescence (3-10). The network of valine, glycine and calcium oxalate enables the formation of aggregates, resulting in the appearance of breathing difficulties (2,9-10). To halt or minimize the intake of valine and glycine and alleviate symptoms, various food regimens can be adopted (1-2,11-13). In order to contain the pandemic, multiple episodes of 1 to 3 days of simultaneous fasting with modest boiled rice water or fruit juice can be implemented, separated by pure starch-based diet or rice starch-based diet with modest fruit juice intake. Rice starch possesses lowest content of proteins among the protein-poor grains. The consumption of pure starch does not give rise to essential amino acids such as valine, which generates bottlenecks in the synthesis of virulence proteins. The duration of fasting or choice of food regimens need to be determined via clinical trials and validated.

\section{Acknowledgement}

This work was supported by grants from the Science and Technology Transformation Program of Sun Yat-sen University of China (33000-18843234) and Guangzhou Science and Technology Program (201804010328) to Q. Liu. We thank Yan Shi for editing.

\section{References}

1. Yan S, Zhang Y, Liu Q. Why COVID-19 virus is so deadly to cancer patients? Eur J Cancer Prev. 2020;29(4):365.

2. Wan Y, Yan S, Zhang Y, An S, Yang K, Xu H, Gan T, et al. The Pneumonia outbreak: High isoleucine and high valine plus glycine contents are features of the proteins of COVID-19 virus. Preprints. 2020. doi: 10.20944/preprints202002.0289.v5

3. Wang Y, Tang JW, Ma WQ, Feng J, Feng J. Dietary zinc glycine chelate on growth performance, tissue mineral concentrations, and serum enzyme activity in weanling piglets. Biol Trace Elem Res 2010;133(3):325-34. https://doi.org/10.1007/s12011-009-8437-3

4. Strasdeit H, Büsching I, Behrends S, Saak W, Barklage W. Syntheses and properties of zinc and calcium complexes of valinate and isovalinate: metal alpha-amino acidates as possible constituents of the early Earth's chemical inventory. Chemistry. 2001;7:1133-42.

5. Olsson J, Hahn RG. Glycine toxicity after high-dose i.v. infusion of $1.5 \%$ glycine in the mouse. Br J Anaesth 1999; 82:250-254.

6. Du HN, Tang L, Luo XY, Li HT, Hu J, Zhou JW, et al. A peptide motif consisting of glycine, alanine, and valine is required for the fibrillization and cytotoxicity of human alpha-synuclein. Biochem. 2003;42(29):8870-8878. 
7. Wan Y, Zhang J, Li X, Wang Y, Liu Q. Cellular states and secondary chemical bonding: a biochemical view of major human diseases. Biochem Insights. 2019;12:1178626419877846.

8. Qi J, Zhang X, Chen Z, Shi Y, Liu Q. Transgenic proteins rich in valine or glycine are concerns for heart disease patients. Eur J Prev Cardiol. 2018;25(8):883-4.

9. An S, Zhang X, Shi Y, Zhang J, Wan Y, Wang Y, et al. High glycine content in TDP-43: a potential culprit in limbic-predominant age-related TDP-43 encephalopathy. J Inter Med Res. 2020;48(6):300060520929853.

10. Zhang X, Ma X, Gan T, Shi Y, Wang Y, Liu Q. Secondary chemical bonding between insoluble calcium oxalate and carbonyl oxygen atoms of GLY and VAL residues triggers the formation of $A \beta$ Aggregates and their deposition in the brain. ACS Chem Neurosci. 2020;11(24):4007-11.

11. Gan T, Fu M, Wu J, Wen L, Liu Q. How to design carbohydrate diet regimens for heart disease patients. Eur J Prev Cardiol. 2018;25(9): 979-80.

12. He R, He S, Li X, Shi Y, Liu Q. How can heart disease patients prevent complications from viral infections? Eur J Prev Cardiol. 2018;25(7):758.

13. Zhou Y, Shi Y, Wan Y, Ye ZW, Liu Q. Why the Mediterranean diet lowers the risk of heart disease. Eur J Prev Cardiol. 2017;24:1788-9.

14. Ouyang, J. L., Fu, M., Peng, J., Tang, M., \& Liu, Q. (2022, February 9). Can starch-based diet or sugar water put an end to the COVID-19 pandemic? OSF Preprints. 2022. osf.io/shmk3. https://osf.io/shmk3/

15. Lin, Y., Liao, L., Lam C., Ouyang, J. L., Fu, M., Tang, M., \& Liu, Q*. (2021, May 4). Starch-based diet, plant-based diet or fasting may be beneficial to individuals suffering from sequelae of SARS-CoV-2 infection. OSF Preprints. May 4. osf.io/svbfy. https://osf.io/svbfy/

16. Chanyuk Lam, Lin, Y., Liao, L., Tang, M., Fu, M., \& Liu, Q*. (2021, April 17). Simultaneous fasting may contain SARS-CoV-2 infection. OSF Preprints. April 17. osf.io/zn9rg. https://osf.io/zn9rg

17. Ying Zhang, Tao Gan, Xiaoxiao Zhang, Qiuyun Liu*. Is lysine supplement beneficial to severe COVID-19 patients? Science, 2020. https://www.science.org/doi/10.1126/science.abb7269

18. Liao L, Zhang J, Wang Y, Liu Q. Why Omicron Variant of SARS-CoV-2 is Less Fatal?. Chembiochem. 2022;23(13):e202200158. doi:10.1002/cbic.202200158

19. Lin, Y., Liao, L., Lam C., Ouyang, J. L., Fu, M., Tang, M., \& Liu, Q. (2021, May 10). The integration of lysine supplement and weak organic acids may hold the key to a longer lifespan. OSF Preprints. May 10. osf.io/r8wby. 